\title{
Measuring Electronic and Structural Transformations in Solar Thermochemical Water Splitting Materials with Aberration-Corrected STEM-EELS
}

\author{
Jamie Trindell $^{1}$, Joshua Sugar ${ }^{2}$ and Anthony McDaniel ${ }^{3}$
}

${ }^{1}$ Sandia National Lab, Pleasanton, California, United States, ${ }^{2}$ Sandia National Laboratories, Livermore, California, United States, ${ }^{3}$ Sandia National Laboratories, United States

Solar thermochemical hydrogen $(\mathrm{STCH})$ production is currently being investigated in the push towards a clean energy infrastructure.1, 2 The STCH process uses solar energy to drive the necessary redox reactions for water-splitting. A STCH cycle consists of a thermal reduction step, releasing O2, and an oxidation or water-splitting step, releasing $\mathrm{H} 2$. In order to understand the rate-limiting and mechanistic details of this process, it is necessary to investigate (1) how the electrons are being transported through the active material during redox cycling and (2) the effects of any structural rearrangements, including defect formations or phase changes, on water-splitting activity. Transition-metal-based perovskites are frequently reported as active STCH cycle materials.3-7 Atomic substitution enables the composition of these perovskites to be varied, resulting in a range of compositions to be explored and discovered as increasingly active STCH materials. The electronic and structural changes of novel ABO3-type perovskites during STCH cycling are examined here. We combine STEM-EELS, electron diffraction analysis, and atomic-resolution STEM imaging to understand the effects of STCH cycling on perovskite structural stability and long-term activity. We apply STEM-EELS to both the oxidized and reduced states of these materials to determine the valence state changes of the A- and B-site ions during the redox reactions.8, 9 In addition, in situ heating in the microscope column vacuum allows us to view the progressive, temperature-dependent chemical changes during reduction, providing mechanistic insight into the redox chemistry of these materials. During the thermal reduction, or activation step, oxygen gas is spontaneously released from the crystalline lattice, forming oxygen vacancies. The resulting strain can lead to the formation of crystalline defects or even complete phase transitions. 3 The combination of diffraction analysis and atomic resolution imaging allows us to correlate the crystalline defect formations with the electronic changes that are measured with EELS. Here, we will compare the water-splitting activity of different perovskite-based STCH materials in correlation with the corresponding microstructural and electronic structure evolution observed during redox cycling. The combination of multiple electron-microscopy based techniques provides valuable nanoscale insight into mechanistic details of the redox reactions that are not obvious when bulk techniques that average measurements over larger volumes are used. Sandia National Laboratories is a multimission laboratory managed and operated by National Technology \& Engineering Solutions of Sandia, LLC, a wholly owned subsidiary of Honeywell International Inc., for the U.S. Department of Energy's National Nuclear Security Administration under contract DE-NA0003525. This paper describes objective technical results and analysis. Any subjective views or opinions that might be expressed in the paper do not necessarily represent the views of the U.S. Department of Energy or the United States Government.

\section{References}

1. Bulfin, B.; Vieten, J.; Agrafiotis, C.; Roeb, M.; Sattler, C., Applications and limitations of two step metal oxide thermochemical redox cycles; a review. Journal of Materials Chemistry A 2017, 5 (36), 18951-18966. 
2. Bayon, A.; de la Calle, A.; Ghose, K. K.; Page, A.; McNaughton, R., Experimental, computational and thermodynamic studies in perovskites metal oxides for thermochemical fuel production: A review. International Journal of Hydrogen Energy 2020, 45 (23), 12653-12679.

3. Barcellos, D. H.; Sanders, M. D.; Tong, J.; McDaniel, A. H.; O’Hayre, R. P., BaCe0.25Mn0.75O3- $\delta-$ a promising perovskite-type oxide for solar thermochemical hydrogen production. Energy \& Environmental Science 2018, 11 (11), 3256-3265.

4. Naghavi, S. S.; He, J. G.; Wolverton, C., CeTi2O6-A Promising Oxide for Solar Thermochemical Hydrogen Production. Acs Applied Materials \& Interfaces 2020, 12 (19), 21521-21527.

5. Qian, X.; He, J.; Mastronardo, E.; Baldassarri, B.; Wolverton, C.; Haile, S. M., Favorable Redox Thermodynamics of SrTi0.5Mn0.5O3- $\delta$ in Solar Thermochemical Water Splitting. Chemistry of Materials 2020, 32 (21), 9335-9346.

6. Haeussler, A.; Abanades, S.; Jouannaux, J.; Julbe, A., Non-Stoichiometric Redox Active Perovskite Materials for Solar Thermochemical Fuel Production: A Review. Catalysts 2018, 8 (12).

7. Dey, S.; Naidu, B. S.; Govindaraj, A.; Rao, C. N., Noteworthy performance of La(1-x)Ca(x)MnO3 perovskites in generating $\mathrm{H} 2$ and $\mathrm{CO}$ by the thermochemical splitting of $\mathrm{H} 2 \mathrm{O}$ and $\mathrm{CO}$. Phys Chem Chem Phys 2015, 17 (1), 122-5.

8. Pearson, D. H.; Fultz, B.; Ahn, C. C., Measurements of 3dstate occupancy in transition metals using electron energy loss spectrometry. Applied Physics Letters 1988, 53 (15), 1405-1407.

9. Yedra, L.; Xuriguera, E.; Estrader, M.; Lopez-Ortega, A.; Baro, M. D.; Nogues, J.; Roldan, M.; Varela, M.; Estrade, S.; Peiro, F., Oxide Wizard: an EELS application to characterize the white lines of transition metal edges. Microsc Microanal 2014, 20 (3), 698-705. 